\title{
Desde el ISET al InDRE. IV. Instituto de Diagnóstico y Referencia Epidemiológicos: nueva orientación, 1990-2012
}

M. del Carmen Guzmán-Bracho, ${ }^{1}$ Jorge A. Ramírez-Hernández,, ${ }^{2 *}$ Martha E. Rodríguez-Pérez, ${ }^{3}$ Carlos Viesca-Treviño ${ }^{3}$ y José A. Díaz-Quiñonez²

${ }^{1}$ Instituto de Diagnóstico y Referencia Epidemiológicos "Dr. Manuel Martínez Báez"; ${ }^{2}$ Universidad Nacional Autónoma de México, Facultad de Medicina, División de Estudios de Posgrado; '3niversidad Nacional Autónoma de México, Facultad de Medicina, Departamento de Historia y Filosofía de la Medicina. Ciudad de México, México

\section{Resumen}

De 1990 a 2012, el Instituto de Salubridad y Enfermedades Tropicales experimentó los cambios más importantes. En 1989 modificó su nombre y orientación a Instituto Nacional de Diagnóstico y Referencia Epidemiológicos. Poco antes se había formalizado como cúspide de la Red Nacional de Laboratorios en Salud Pública y había incorporado los laboratorios de programas preventivos como el de citología exfoliativa y los de diagnóstico de rabia, paludismo, tuberculosis; posteriormente incorporaría otras redes que surgieron como parte de la respuesta a brotes epidémicos y al nuevo panorama epidemiológico. En este periodo, 27 algoritmos diagnósticos se definieron y organizaron en 18 redes, algunas de las cuales comenzaron a colaborar con redes globales. En 2001, en el Instituto se empezó a trabajar con patógenos relacionados con el bioterrorismo. Para entonces, las restricciones del edificio sede fueron evidentes; en 2008, se decidió construir nuevas instalaciones. El Instituto y sus redes diagnósticas constituyen un hito en la salud pública latinoamericana del siglo XXI.

PALABRAS CLAVE: Instituto de Diagnóstico y Referencia Epidemiológicos. Salud pública. Historia de la medicina.

\section{From ISET to InDRE. IV. Institute of Epidemiological Diagnosis and Reference: new orientation, 1990-2012}

\section{Abstract}

From 1990 to 2012, the Sanitary and Tropical Diseases Institute experienced the most important changes. In 1989, its name and orientation were modified to become the National Institute of Epidemiological Diagnosis and Reference (InDRE). Shortly before, it had been formalized as the apex of the National Network of Public Health Laboratories and had incorporated laboratories for preventive programs such as exfoliative cytology and rabies, malaria and tuberculosis diagnosis; subsequently, it would incorporate other networks that emerged as part of the response to major epidemic outbreaks and to the new epidemiological outlook. In this period, 27 priority diagnostic algorithms were defined and organized in 18 networks, some of which began to collaborate with global networks. In 2001, the Institute started working with pathogens related to bioterrorism. By then, space restrictions of the headquarters' building were evident; in 2008, starting the construction of new facilities was decided. The institute and its diagnostic networks constitute a milestone in Latin American public health of the 21 st century.

KEY WORDS: Institute of Epidemiological Diagnosis and Reference. Public health. History of medicine.

Correspondencia:

*Jorge A. Ramírez-Hernández

E-mail: jorgealejandrorh @ hotmail.com

DOI: 10.24875/GMM.20005564

0016-3813/@ 2020 Academia Nacional de Medicina de México, A.C. Publicado por Permanyer. Este es un artículo open access bajo la licencia CC BY-NC-ND (http://creativecommons.org/licenses/by-nc-nd/4.0/).
Fecha de recepción: 01-10-2019

Fecha de aceptación: 03-01-2020 


\section{Introducción}

Este artículo cubre el periodo 1990-2012 del Instituto de Salubridad y Enfermedades Tropicales (ISET) que se transformó en el Instituto Nacional de Diagnóstico y Referencia Epidemiológicos. Se cambió el nombre durante el primer trimestre de 1989 y se fortaleció la colaboración entre la epidemiología y los programas preventivos a través de la Red Nacional de Laboratorios de Salud Pública (RNLSP), iniciada en el ISET en 1985. ${ }^{1-3}$ Se desarrolló la interacción del InDRE con laboratorios de referencia internacional y redes globales de diagnóstico. En esa RNLSP también participaba el Laboratorio Nacional de Salud Pública (ahora Comisión de Control Analítico y Ampliación de Cobertura) como referencia para la vigilancia sanitaria de lácteos, agua y alimentos. ${ }^{4}$

En 1997 y 2010, el INDRE y la RNLSP obtuvieron el respaldo normativo. ${ }^{5,6}$ En 2000 , derivado de la Ley de los Institutos Nacionales de Salud y sin alterar su nueva orientación, en el nombre del Instituto se eliminó el término "Nacional" para quedar como Instituto de Diagnóstico y Referencia Epidemiológicos (InDRE). ${ }^{7}$ Solo se mencionará esta denominación.

En el periodo de estudio, el InDRE asumió la responsabilidad como laboratorio de referencia con base en los criterios de la Organización Mundial de la Salud: laboratorio gubernamental especializado que analiza a profundidad las muestras recibidas; asesora, prepara, estandariza o evalúa reactivos para diagnóstico; emite directrices para la operación de otros laboratorios, capacita, realiza investigación y mantiene relación con centros internacionales análogos. ${ }^{8}$

\section{Encuesta Nacional Seroepidemiológica, un reto superado}

La Encuesta Nacional Seroepidemiológica exigió al ISET desarrollar infraestructura y capacidades para almacenar, distribuir y procesar medio millón de sueros con su información epidemiológica. Los procedimientos se realizaron con métodos estándar, bajo el control de laboratorios de referencia internacional. ${ }^{9}$ Los resultados, publicados en 1992, establecieron la línea base de esos padecimientos en México (Tabla 1).

\section{La situación internacional}

La transición epidemiológica y los nuevos retos globales posicionaron al laboratorio de salud pública.
En la década de 1990 se efectuaron iniciativas globales como la Declaración de Río sobre el Medio Ambiente y el Desarrollo (1992). El Banco Mundial presentó Invirtiendo en salud, informe cuyo objetivo era coadyuvar a mejorar la eficiencia del sector en países de ingreso medio y bajo (1993). La iniciativa Metas del Milenio (1995), de las Naciones Unidas, señaló como prioritarios el $\mathrm{VIH} /$ sida, la tuberculosis, la enfermedad de Chagas y el paludismo. Poco después, la Organización Mundial de la Salud hizo un balance de la situación epidemiológica global, aún caracterizada por alta mortalidad atribuible a enfermedades infecciosas. Para fines del siglo XX reconoció la importancia de las enfermedades crónico-degenerativas y desarrolló el Convenio Marco para el Control del Tabaco. Por su parte, la Federación Mundial del Corazón llamó la atención sobre el incremento de padecimientos cardiovasculares en países de América Latina y Europa Oriental. En 2002, la Organización Mundial de la Salud inició la Estrategia Mundial sobre Régimen Alimentario, Actividad Física y Salud. En ese año surgió el Fondo Mundial para la Lucha contra el $\mathrm{VIH} / \mathrm{sida}$, la Tuberculosis y la Malaria; algunos recursos llegarían al InDRE.

\section{La RNLSP para el diagnóstico epidemiológico. Organización y proceso de integración}

\section{Adolfo Pérez Miravete escribió:10}

\begin{abstract}
[En 1985]...se revisó el papel que la institución podía jugar en la nueva organización de la Secretaría, decidiendo darle una nueva orientación con el propósito de llenar algunas necesidades percibidas en los servicios epidemiológicos, particularmente como infraestructura de los servicios de vigilancia de enfermedades transmisibles. La organización de la Red Nacional de Laboratorios de Salud [sic] también requería una institución nacional situada en la cúspide de la organización piramidal.
\end{abstract}

Con fondos del Programa de Naciones Unidas para el Desarrollo, ${ }^{10}$ entre 1985 y 1989 se reorganizaron las responsabilidades de la RNLSP,11 cuya disposición se muestra en la Figura 1, en la cual las líneas verticales de la cara frontal de la pirámide representan las redes de laboratorios para diagnósticos obligatorios para los 32 laboratorios estatales de salud pública (LESP). La cara lateral muestra los diagnósticos no organizados en red ni obligatorios que se realizan en el InDRE con apoyo de otros niveles. En el esquema, los procesos de apoyo y administración están representados por líneas horizontales. Cuando se cruzan con los procedimientos técnicos (líneas verticales) 
Tabla 1. Encuesta Nacional Seroepidemiológica. Diagnósticos efectuados en el ISET de 1987 a 1989

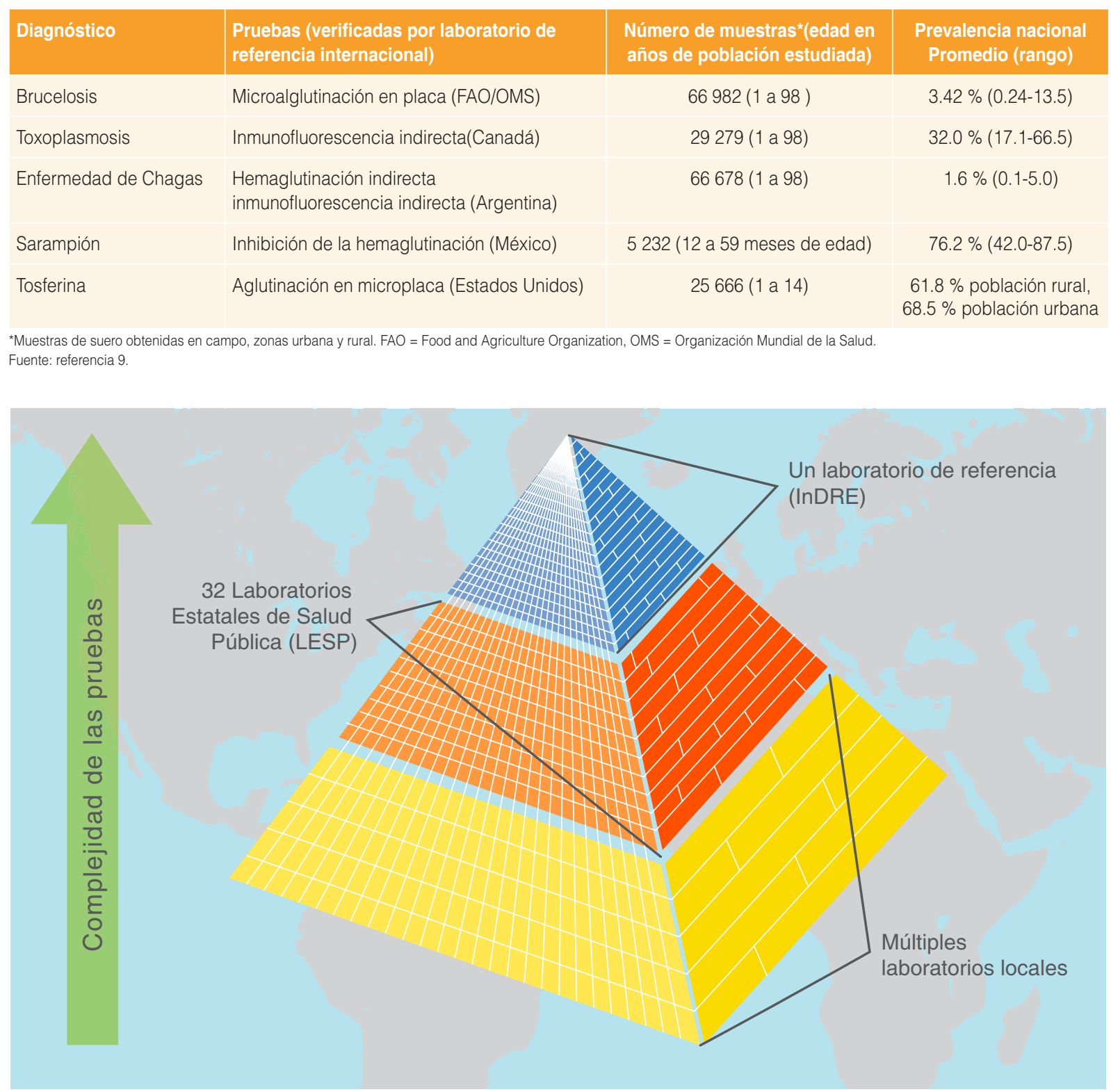

Figura 1. Organización piramidal de la Red Nacional de Laboratorios de Salud Pública. Diseño: Francisco Meza Gordillo.

producen un entramado con cientos de puntos de control.

Para 1992, la RNLSP ya estaba estructurada en tres niveles: el federal a cargo del InDRE, el estatal con los LESP y el local. Estaba integrada por casi 900 laboratorios organizados por diagnóstico. Al término del siglo XX, la RNLSP contaba con 19 LESP y cuatro más estaban en construcción. ${ }^{12}$ Cada grupo de laboratorios que realizaban el mismo diagnóstico obligatorio integraron una red específica: red de paludismo, red de tuberculosis, etcétera (Tabla 2).

Es interesante el proceso de integración seguido por la RNLSP. De 1947 a 1984, el diagnóstico citológico se realizaba en unidades médicas en un modelo asistencial..$^{13}$ En la década de 1970, el diagnóstico de rabia se inició en el Laboratorio Regional de Virología, después Laboratorio Estatal de Salud Pública en el Estado de México (comunicación personal de Nidia Aréchiga). Los laboratorios de los programas de paludismo y tuberculosis trabajaban por separado. Todos se integraron a la RNLSP en la década de 1980 (Tabla 2).

En los años siguientes, el programa de paludismo recibía información oportuna proveniente de aproximadamente 250 microscopistas estatales y locales (comunicación personal de Sonia Galindo) y de la red 
Tabla 2. Red Nacional de Laboratorios de Salud Pública en 1992

\begin{tabular}{|c|c|c|c|c|}
\hline \multirow[t]{2}{*}{ Función principal } & Laboratorio central & Laboratorios estatales (n) & Laboratorios locales (n) & \multirow{2}{*}{$\begin{array}{c}\text { Entidades federativas } \\
\text { participantes (n) }\end{array}$} \\
\hline & Coordinación y referencia & Diagnóstico y enlace & Diagnóstico y tamizaje & \\
\hline \multicolumn{5}{|l|}{ Diagnóstico } \\
\hline Paludismo & InDRE & 3 & 138 & 27 \\
\hline Tuberculosis y lepra & InDRE & 10 & 474 & 32 \\
\hline Cáncer cervicouterino & InDRE & 2 & 40 & 16 \\
\hline Rabia & InDRE & 10 & 6 & 13 \\
\hline VIH/sida & InDRE & 6 LES y 17 CETS & 86 & 32 \\
\hline Cólera & InDRE & 16 & 73 & 31 \\
\hline $\begin{array}{l}\text { Histocompatibilidad } \\
\text { para trasplantes }\end{array}$ & InDRE & 1 & 23 & 9 \\
\hline
\end{tabular}

de entomología, la cual también reforzó la vigilancia de otros padecimientos transmitidos por vector. El programa de tuberculosis fue apoyado por 31 LESP y más de 700 laboratorios locales (comunicación personal de Susana Balandrano).

Otro mecanismo fue la creación de redes como respuesta ante algunos padecimientos: la Red Nacional de VIH en 1986, la Red Nacional de Cólera en $1991^{14}$ y la Red de Infecciones Respiratorias Agudas Bacterianas en 2002 (comunicación personal de Luis Ángel Sapian López e Isabel Moreno Camilli) (Tabla 2).

Las redes se mantenían en constante evolución. Dos ejemplos: el diagnóstico de enfermedad febril exantemática incluía sarampión, rubeola y dengue; la relevancia de este último motivó la creación de una red específica para dengue, que incluyó otras arbovirosis existentes en México o en riesgo de introducirse. Desde 2009, la Red de Influenza y otros Virus Respiratorios empleó metodologías moleculares en Ios LESP y cinco laboratorios de apoyo a la vigilancia epidemiológica, pertenecientes a distintas instituciones de salud: Instituto Mexicano del Seguro Social, Instituto de Seguridad y Servicios Sociales de los Trabajadores del Estado, Hospital General, Instituto Nacional de Ciencias Médicas y Nutrición e Instituto Nacional de Enfermedades Respiratorias (Tabla 3).

El estrecho seguimiento del desempeño de cada LESP y la implementación de sistemas de gestión de calidad y bioseguridad a partir de 2002 (comunicación personal de Celia González Bonilla y Lucía Hernández) proporcionaron un marco de oportunidad y confiabilidad al desarrollo de la RNLSP. ${ }^{15}$

Con la finalidad de armonizar el trabajo del laboratorio con la epidemiología se definieron 27 algoritmos diagnósticos prioritarios, organizados en 18 redes de diagnóstico, en las que debían participar todos los
LESP (comunicación personal de Tere Martín Escobar) (Tabla 3).

En la década de 1990, en el InDRE hubo intensa producción de reactivos e insumos que aseguró el diagnóstico en los LESP. Se introdujeron metodologías para estudios seroepidemiológicos a gran escala (comunicación personal de Herlinda García Lozano), técnicas moleculares para identificar y caracterizar agentes, ${ }^{16}$ así como la evaluación de la aplicabilidad en población mexicana de pruebas comerciales disponibles (comunicación personal de Roberto Vázquez Campuzano), que respaldó el carácter anticipatorio de la vigilancia. ${ }^{17}$

El InDRE implementó más de 300 algoritmos propios, entre ellos los de poliomelitis, micosis, cisticercosis, amibas de vida libre y patógenos involucrados en bioterrorismo, así como procedimientos para determinar la resistencia a drogas antivirales y antibacterianas y la producción de reactivos biológicos.

\section{Flujo de muestras e información. Cadena de custodia y bioseguridad}

El pulso y la capilaridad de la organización piramidal, inadvertidos para el usuario externo, son resultado del esfuerzo de miles de trabajadores que movilizan la información clínico-epidemiológica con las muestras (tejido y fluido humanos, el patógeno mismo, espécimen entomológico o material genético), consideradas de alto valor epidemiológico. Cada muestra es tomada, identificada, conservada, embalada, transportada desde cualquier localidad hasta su lugar de procesamiento y resguardo (o disposición) final en algún laboratorio de la red. Este proceso dinámico tiene dos elementos: el cuidado de la muestra con su información asociada (cadena de custodia) y la protección de profesionales, población y medio ambiente (bioseguridad). 
Tabla 3. Red Nacional de Laboratorios de Salud Pública

\begin{tabular}{|c|c|c|c|c|}
\hline \multicolumn{2}{|c|}{ Algoritmo diagnóstico* } & \multirow{2}{*}{$\begin{array}{l}\text { Padecimientos }{ }^{\dagger} \\
\text { Cáncer del cuello del útero }\end{array}$} & \multirow{2}{*}{$\begin{array}{l}\text { Inicio de la red } \\
\text { en México } \\
\qquad 1947^{\ddagger}\end{array}$} & \multirow{2}{*}{$\begin{array}{l}\text { Inicio del diagnóstico } \\
\text { en el ISET-InDRE } \\
1985\end{array}$} \\
\hline 1 & Citología del cuello uterino & & & \\
\hline 2 & Paludismo & $\begin{array}{l}\text { Paludismo por Plasmodium vivax (autóctono), } \\
\text { falciparum, malariae y ovale (importados), enfermedad } \\
\text { de Chagas aguda, leishmaniasis cutánea }\end{array}$ & $1955^{\ddagger}$ & 1984 \\
\hline 3 & Tuberculosis & $\begin{array}{l}\text { Tuberculosis pulmonar, extrapulmonar y } \\
\text { farmacorresistente }\end{array}$ & $1971^{\ddagger}$ & 1987 \\
\hline 6 & Rabia & Rabia humana y en animales domésticos y silvestres & 1972 & 1979 \\
\hline 4 & $\mathrm{VIH} / \mathrm{sida}^{* \star}$ & Infección por VIH/sida & 1986 & 1985 \\
\hline 5 & Entomología & $\begin{array}{l}\text { Enfermedades transmitidas por mosquitos vectores, } \\
\text { chinches hematófagas, artrópodos ponzoñosos, } \\
\text { ectoparásitos y otros taxones }\end{array}$ & 1987 & 1939 \\
\hline 7 & $\begin{array}{l}\text { Enfermedad diarreica aguda } \\
\text { bacteriana }\end{array}$ & $\begin{array}{l}\text { Cólera, brotes de gastroenteritis por Vibrio } \\
\text { parahemolyticus y Escherichia coli, salmonelosis, } \\
\text { shigelosis }\end{array}$ & 1991 & 1939 \\
\hline 8 & Brucelosis & Brucelosis humana & 1992 & 1939 \\
\hline 9 & $\begin{array}{l}\text { Enfermedad febril } \\
\text { exantemática }\end{array}$ & $\begin{array}{l}\text { Sarampión, rubeola, rubeola congénita, diagnóstico } \\
\text { diferencial por parotiditis, varicela, infecciones por } \\
\text { virus de Epstein-Barr, parvovirus B19 y otros virus } \\
\text { exantemáticos }\end{array}$ & 1992 & 1972 \\
\hline 10 & Enfermedad de Chagas & Enfermedad de Chagas aguda, congénita o crónica & 1994 & 1939 \\
\hline 11 & Leishmaniasis & $\begin{array}{l}\text { Leishmaniasis cutánea, mucocutánea, cutánea difusa } \\
\text { y visceral }\end{array}$ & 1994 & 1939 \\
\hline 12 & Tosferina & Tos ferina y síndrome coqueluchoide & 1995 & 1942 \\
\hline 13 & Dengue y otras arbovirosis ${ }^{\dagger+}$ & $\begin{array}{l}\text { Dengue, zika, chikunguña, enfermedad por el virus del } \\
\text { Nilo occidental, fiebre amarilla }\end{array}$ & 1995 & 1978 \\
\hline 14 & Rotavirus y otros enterovirus & $\begin{array}{l}\text { Gastroenteritis por rotavirus, norovirus, sapovirus, } \\
\text { astrovirus, adenovirus entéricos } 40 \text { y } 41\end{array}$ & 1996 & 1994 \\
\hline 15 & $\begin{array}{l}\text { Influenza y otros virus } \\
\text { respiratorios }\end{array}$ & $\begin{array}{l}\text { Influenza e infecciones por virus sincicial respiratorio, } \\
\text { metapneumovirus humano, parainfluenza 1, 2, } 3 \text { y 4; } \\
\text { coronavirus 229E, OC43, HKU1, NL63, adenovirus, } \\
\text { rhinovirus, enterovirus y bocavirus }\end{array}$ & 1997 & 1951 \\
\hline 16 & Leptospira & Leptospirosis humana & 1997 & 1993 \\
\hline 17 & Hepatitis & Hepatitis A, B, C & 1998 & 1973 \\
\hline 18 & $\begin{array}{l}\text { Infecciones respiratorias } \\
\text { agudas bacterianas }\end{array}$ & $\begin{array}{l}\text { Infecciones respiratorias agudas graves e infecciones } \\
\text { invasivas causadas por Streptococcus pneumoniae, } \\
\text { Neisseria meningitidis y Haemophilus influenzae }\end{array}$ & 2002 & 1980 \\
\hline 19 & Rickettsiosis & $\begin{array}{l}\text { Fiebre manchada de las Montañas Rocosas, tifus } \\
\text { epidémico, tifus murino o endémico }\end{array}$ & 2016 & 1973 \\
\hline 20 & $\begin{array}{l}\text { Infecciones de transmisión } \\
\text { sexual|‡ }\end{array}$ & $\begin{array}{l}\text { Sífilis, herpes genital, linfogranuloma venéreo, uretritis y } \\
\text { cervicitis gonocócica, uretritis no gonocócica, cervicitis } \\
\text { mucopurulenta, flujo vaginal, enfermedad pélvica } \\
\text { inflamatoria, virus del papiloma humano }\end{array}$ & 2016 & 1974 \\
\hline HInic & $\begin{array}{l}\text { los nombres vigentes en 2019. Cada } \\
\text { nan los indicados en los algoritmos di } \\
\text { os coordinados por los programas pre } \\
\text { ada de 1970, el laboratorio de referen } \\
\text { des de Transmisión Sexual. } \\
\text { vidades en la Red de Enfermedad Fet }\end{array}$ & $\begin{array}{l}\text { itmo diagnóstico constituye una red de laboratorio } \\
\text { sticos vigentes en } 2019 \text {. } \\
\text { vos y de control. El Laboratorio de Control de Calidad de Cáncer Cérvico } \\
\text { el Centro Eliseo Ramírez para infecciones de transmisión sexual se integr } \\
\text { kantemática. }\end{array}$ & $\begin{array}{l}\text { lo se inició hasta } \\
\text { ET. La red se inic }\end{array}$ & o Red de VIH y otras \\
\hline
\end{tabular}

"Inició actividades en la Red de Enfermedad Febril Exantemática.

抽uncionó desde 1985 hasta 2016 dentro de la Red de VIH y otras Enfermedades de Transmisión Sexual. 
Los materiales biológicos resguardados en los bancos de muestras, algunos conservados desde hace ocho décadas, son un invaluable acervo para la investigación y la referencia.

\section{La respuesta del InDRE a eventos de enfermedades infecciosas}

Algunos eventos epidémicos (como los del cólera, $\mathrm{VIH} /$ sida y dengue) motivaron la creación de redes. Es importante destacar la respuesta especial dada al sarampión y a la pandemia de influenza. ${ }^{18-22}$

La atención del brote de cólera en 1991 resultó emblemática por la capacitación que los profesionales del InDRE realizaron en cascada a los LESP, Ios cuales la reprodujeron a 239 laboratorios locales, públicos y privados. ${ }^{23}$ El InDRE efectuó la genotipificación y susceptibilidad antimicrobiana, elaboró y envió reactivos biológicos a los LESP, supervisó y evaluó el tamizaje estatal. ${ }^{24}$

Este exitoso modelo de transferencia de tecnología sería utilizado para enfrentar brotes y emergencias sanitarias por dengue, cólera y leptospirosis en eventos hidrometeorológicos, incluso para apoyar a países centroamericanos.

En 2001 se estableció el diagnóstico de ántrax como preparación ante potenciales acciones de bioterrorismo; se proporcionó evidencia del bajo riesgo para la población mexicana (comunicación personal de Hiram Olivera Díaz). Se fortaleció la gestión del riesgo biológico en la RNLSP.

La influenza $A(\mathrm{H} 1 \mathrm{N1})$ pdm llegó a México cuando los métodos clásicos de virología y biología molecular estaban suficientemente desarrollados para realizar confirmación, subtipificación, aislamiento, secuenciación, análisis bioinformáticos y vigilancia de resistencia a antivirales (comunicación personal de Ernesto Ramírez González e Irma López). El InDRE desplegó una respuesta rápida -inicialmente durante las 24 horas de todos los días de la semana- y transfirió técnicas moleculares en tiempo real que agilizaron la respuesta y la evaluación del impacto poblacional. La respuesta especial ante la pandemia (Sistema del Comando de Incidentes) incluyó a toda la RNLSP. ${ }^{25,26}$

\section{Bioterrorismo, alerta temprana y contención}

Desde 1999, el InDRE participa oficialmente en la Convención sobre la Prohibición del Desarrollo, la Producción y el Almacenamiento de Armas Bacteriológicas y Toxínicas y sobre su Destrucción (comunicación personal de Irma Hernández-Monroy).

En 2001, además del diagnóstico del virus del oeste del Nilo, SARS Co-V y ántrax, en el Instituto se añadió la capacidad diagnóstica para Francisella tularensis, Yersinia pestis, variola virus y toxina botulínica, ${ }^{27-29}$ ante el riesgo de una liberación intencionada. ${ }^{30}$

Ese mismo año, México se incorporó a la Iniciativa Global para la Seguridad en Salud. En la red de laboratorios de esta iniciativa participa el InDRE, en el G7+México, lo que le permitió mantener capacidades para atender amenazas a la seguridad nacional en materia de salud.

En 2010, en Estados Unidos se reconoció el esfuerzo del InDRE, el cual fue incluido en la Red de Laboratorios de Respuesta (LRN, Laboratory Response Network), una selecta red de laboratorios en la que participan los Centros de Control y Prevención de Enfermedades (CDC), el Departamento de Salud (DHHS), el Departamento de Agricultura (USDA), la Agencia de Alimentos y Medicamentos (FDA), el Buró Federal de Investigaciones (FBI) y otras agencias estadounidenses.

Solo Canadá, Australia, Reino Unido y México son países miembros de la LRN y mantienen procedimientos estándar de laboratorio para responder a eventos de bioterrorismo, enfermedades infecciosas emergentes, terrorismo químico y otras contingencias de salud pública.

\section{Banco de material biológico de referencia}

Los materiales biológicos en resguardo institucional (cepas, muestras humanas, material entomológico, secuencias genéticas) y otros adquiridos de bancos internacionales (estándares, controles) son indispensables para alcanzar la alta calidad en el desempeño de un laboratorio de referencia.

Un ejemplo es la Colección de Artrópodos con Importancia Médica, iniciada en 1938 y catalogada desde 1987. La continua incorporación de ejemplares ha hecho posible contar con la colección más grande de Latinoamérica (comunicación personal de Sergio Ibáñez).

El registro, clasificación y embalaje del material biológico para trasladarlo a la nueva sede del InDRE se realizó bajo estrictas condiciones de bioseguridad y biocustodia, con apego a las regulaciones nacionales e internacionales. 


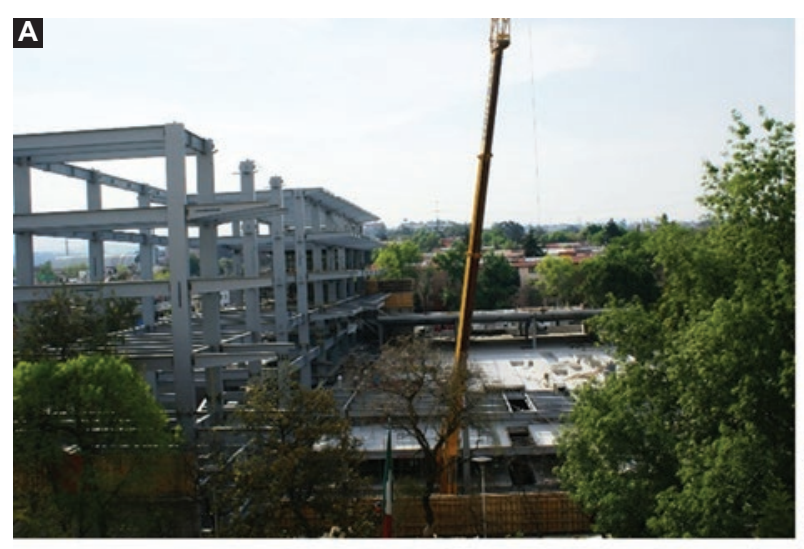

2010

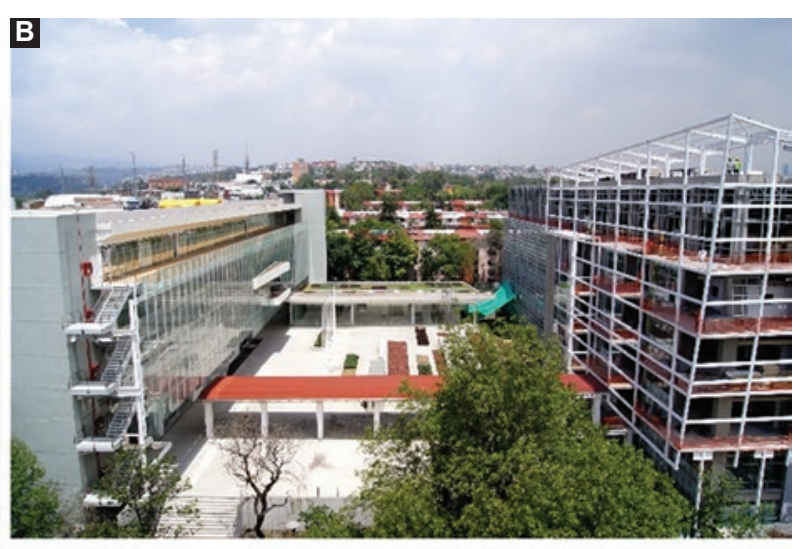

2011

Figura 2. Construcción de las nuevas instalaciones del InDRE en Lomas de Plateros, Ciudad de México. A) Año 2010, foto de Rita Flores León. B) Año 2011, foto de Amelia Patiño González.

Tabla 4. Directores del Instituto de Diagnóstico y Referencia Epidemiológicos, 1990-2019

\begin{tabular}{|l|c|}
\hline Director & Periodo \\
\hline José Luis Valdespino Gómez & $1990-1994$ \\
\hline Ana Flisser Steinbruch & $1995-2000$ \\
\hline Elsa Josefina Sarti Gutiérrez & $2001-2003$ \\
\hline Ignacio Federico Villaseñor Ruiz & $2003-2007$ \\
\hline Celia Mercedes Alpuche Aranda & $2007-2012$ \\
\hline José Alberto Díaz Quiñonez & $2012-2019$ \\
\hline
\end{tabular}

\section{La construcción de las nuevas instalaciones del InDRE}

En 2007, ante la obsolescencia e inseguridad de las instalaciones para los trabajadores y su entorno inmediato, las autoridades de salud reconocieron la necesidad de una nueva y moderna infraestructura para el InDRE. El documento Bases del diseño para las nuevas instalaciones del InDRE, de 2008, hizo notar las condiciones de las instalaciones construidas en 1935.

El proyecto ejecutivo del edificio de laboratorios fue desarrollado por una compañía internacional especializada, con un diseño contemporáneo y materiales y sistemas constructivos que optimizaran la operación $y$ el mantenimiento. ${ }^{31}$ El edificio administrativo fue proyectado en la tradición de la escuela mexicana de arquitectura (Figura 2). La superficie total construida, de casi $17000 \mathrm{~m}^{2}$ de laboratorios, impulsaría el cumplimiento de la misión del InDRE en los ámbitos nacional e internacional (Smith Carter-ICEMEX, InDRE. Diseño esquemático/bases de diseño, 2008).

\section{Discusión}

En 1989, el ISET se transformó en InDRE y dedicó su actividad a la vigilancia y referencia epidemiológicas. El cambio de nombre de la institución, de ISET a InDRE fue un proceso gradual, discreto sin ceremonia, durante el primer trimestre de ese año (comunicación personal de Alejandro Escobar).

En 1985, el ISET ya había sido designado cabeza de la Red Nacional de Laboratorios de Salud Pública, la cual obtuvo respaldo legal en 1997 y 2010. Estas decisiones ayudaron a enfrentar los eventos epidémicos de VIH/sida, cólera, dengue, sarampión e influenza, los cuales requirieron respuesta especial de la RNLSP.

Para finales de este crucial periodo institucional, con la RNLSP en operación pero con nuevos desafíos epidemiológicos como la pandemia de influenza, se hizo evidente que la infraestructura del InDRE era inapropiada. El edificio art déco de 1935 fue reemplazado por instalaciones arquitectónicas de vanguardia, para consolidar el intenso trabajo nacional e impulsar la participación global. Se anexa la lista de directores en el periodo 1990-2019 (Tabla 4).

El InDRE y sus redes diagnósticas constituyen un hito para la salud pública latinoamericana del siglo XXI.

\section{Agradecimientos}

A las autoridades y personal del Archivo Histórico de la Secretaría de Salud, por compartir de manera desinteresada sus conocimientos y por su valiosa orientación en la revisión de los expedientes. 


\section{Bibliografía}

1. Ramírez-Hernández JA, Guzmán-Bracho C, Díaz-Quiñonez JA. Desde el ISET al InDRE. I. Instituto de Salubridad y Enfermedades Tropicales: génesis y primeros años, 1934-1940. Gac Med Mex. 2019;155:322-327.

2. Ramírez-Hernández JA, Guzmán-Bracho MC, Viesca-Treviño C Díaz-Quiñonez JA. Desde el ISET al InDRE. II. Instituto de Salubridad y Enfermedades Tropicales: madurez y consolidación, 1940-1964. Gac Med Mex. 2019;155:398-405.

3. Ramírez-Hernández JA, Guzmán-Bracho C, Rodríguez-Pérez ME, Viesca-Treviño C, Díaz-Quiñonez JA. Desde el ISET al InDRE. III. Instituto de Salubridad y Enfermedades Tropicales: crisis y renovación, 19651989. Gac Med Mex. 2019;155:641-646.

4. Del Río-Zolezzi A, Valdespino-Gómez JL, García-García ML, Giono-Cerezo S, Escobar-Gutiérrez A. La Red Nacional de Laboratorios de Salud Pública en México. Higiene. 1994;2:101-120.

5. Reglamento Interior de la Secretaría de Salud. México: Diario Oficial de la Federación 2001 Jul 5

6. Decreto que reforma, adiciona y deroga diversas disposiciones del Reglamento Interior de la Secretaría de Salud. México: Diario Oficial de la Federación 2010 Feb 2.

7. Flisser-Steinbruch A. Editorial. Salud Publica Mex. 2000;42:482-483.

8. Planificación, organización y administración de un servicio nacional de salud pública. Tercer informe del Comité de Expertos en Servicios de Laboratorio de Salud Pública. Organización Mundial de la Salud; 1962.

9. Sepúlveda-Amor J, Tapia-Conyer R. Encuesta Nacional Seroepidemiológica. Número Especial. Salud Publica Mexico. 1992;34:119-254.

10. Pérez-Miravete, A. Quincuagésimo aniversario del Instituto de Salubridad y Enfermedades Tropicales. Archivo familia Pérez de la Mora.

11. Sepúlveda-Amor J. La vigilancia en salud pública y las Redes Nacionales de Laboratorios. Higiene. 1994;2:95-100.

12. Flisser A, Velasco-Villa A, Martínez-Campos C, González-Domínguez F, Briseño-García B, García-Suárez, et al. Infectious diseases in Mexico. A survey from 1995-2000. Arch Med Res. 2002:33:343-350.

13. Carrillo AM. Entre el 'sano temor' y el 'miedo razonable': la Campaña Nacional Contra el Cáncer en México. Historia Ciencias Saude. 2010;17:89-107.

14. Valdespino-Gómez JL. Instituto Nacional de Diagnóstico y Referencia Epidemiológicos Dr. Manuel Martínez Báez 1992-1993. México: Instituto Nacional de Diagnóstico y Referencia Epidemiológicos "Dr. Manuel Martínez Báez"; 1992.

15. Secretaría de Salud [sitio web]. Red Nacional de Laboratorios de Salud Pública/Instituto de Diagnóstico y Referencia Epidemiológicos. Caminando a la Excelencia. 2003.
16. Escobar-Gutiérrez A, Flisser A. La trascendencia de la metodología molecular en el diagnóstico. Gac Med Mex. 1997;133:105-110.

17. Editorial. Higiene. 1994;2:93-94.

18. Lineamientos para la vigilancia por laboratorio de la infección por el virus de la inmunodeficiencia humana (VIH). México: Instituto de Diagnóstico y Referencia Epidemiológicos “Dr. Manuel Martínez Báez"/Secretaría de Salud; 2017.

19. Lineamientos para la vigilancia por laboratorio de la enfermedad diarreica aguda bacteriana. México: Instituto de Diagnóstico y Referencia Epidemiológicos "Dr. Manuel Martínez Báez"/Secretaría de Salud; 2017.

20. Lineamientos para la vigilancia por laboratorio de la enfermedad febril exantemática. México: Instituto de Diagnóstico y Referencia Epidemiológicos "Dr. Manuel Martínez Báez"/Secretaría de Salud; 2018.

21. Lineamientos para la vigilancia por laboratorio de dengue y otras arbovirosis. México: Instituto de Diagnóstico y Referencia Epidemiológicos "Dr. Manuel Martínez Báez"/Secretaría de Salud; 2017.

22. Lineamientos para la vigilancia por laboratorio de la influenza y otros virus respiratorios. México: Instituto de Diagnóstico y Referencia Epidemiológicos "Dr. Manuel Martínez Báez"/Secretaría de Salud; 2017.

23. Sepúlveda J, Valdespino JL, García-García L. Cholera in Mexico: the paradoxical benefits of the last pandemic. Int J Infect Dis. 2006;10:4-13.

24. Giono-Cerezo S, Rodríguez-Ángeles MG, Gutiérrez-Cogco L, Valdespino-Gómez JL. Caracterización fenotípica y genotípica de Vibrio cholerae O1. Rev Latinoam Microbiol. 1994;36:243-251.

25. Díaz-Quiñonez JA, Alpuche-Aranda CM. Métodos diagnósticos de influenza por laboratorio. En: Córdova-Villalobos, Valdespino-Gómez, Ponce-de León. La epidemia de influenza A/H1N1 en México. México: Editorial Médica Panamericana; 2010.

26. Díaz-Quiñonez JA. Libro blanco 2006-2012. México: Instituto de Diagnóstico y Referencia Epidemiológicos; 2012.

27. Valdespino-Gómez JL, García-García ML. El A, B, C, sobre ántrax, para personal de salud. Salud Publica Mex. 2001;43:604-613.

28. Lineamientos para la vigilancia, prevención y control de enfermedades asociadas a riesgos biológicos. México: Secretaría de Salud; 2001.

29. Sarti E, Moreno-Galván M, Rodríguez-Angeles G, Viveros G, Flores-León R, Tapia-Conyer R. Molecular characterization of anthrax in positive powders: a Mexican experience. J Clin Microbiol. 2003; 41:4909.

30. Franco-Paredes C, Lammoglia L, Santos-Preciado JI. Perspectiva histórica de la viruela en México: aparición, eliminación y riesgo de reaparición por bioterrorismo. Gac Med Mex. 2004;140:321-327.

31. HKS Arquitectos. Laboratorios para el Instituto de Diagnóstico y Referencia Epidemiológicos (InDRE). Rev arquiTK. 2013;12:74-77. 